\title{
A Note on Morphophonological Changes of Fulfulde Verb Suffixes
}

\author{
1,* Bashir Usman, ${ }^{2}$ Idris Muhammad Bello, \& ${ }^{3}$ Adamu Muhammad Abubakar \\ 1,2,3 Languages and Linguistics Department, Faculty of Arts, University of Maiduguri, Borno State, \\ Nigeria
}

Received: 03.01.2020 • Accepted: 01.03.2021 • Published: 31.03.2021 • Final Version 31.03.2021

\begin{abstract}
This paper attempts to analyse the changes of Fulfulde verb suffixes, with particular attention to Adamawa Dialect. The aim of the paper is to analyse verb suffixes to identify the nature of the changes and the conditions that trigger them. The study employed unstructured observation to collect the data and oral interview was used to validate them. Generative Phonology (GP) was adopted as the theoretical framework for data analysis in the study. The study reveals that both phonology and morphology interact in the process of changes that occur within the verb suffixes in Fulfulde. It shows that each voice has a single underlying suffix, which interacts with various tense suffixes to derive a complete verbal complex. Considering the various environments that have different suffixes with common characteristics across the three voices, the changes are found to be triggered by certain rulebased conditions.
\end{abstract}

Keywords: Verb suffixes; Fulfulde; Verbal system.

\section{Introduction}

This paper attempts to explain the changes of suffixes within a verb and considers its forms and behaviours. The main concern is the changes suffixes undergo within verbs of the language. So, other word categories: nouns, pronouns, adverbs, adjectives, etc. will not be discussed here since it is outside the topic of discussion.

Firstly, Fulfulde is an inflectional language largely spoken through the verbs than nouns, most of its words are derived through verbs (Mukoshy, 1977). In general, it has some few primary nouns; most of which are secondary and are derived from verbs which are referred to as verbo-nominals by Arnott (Arnott, 1970). Probably, because of this phenomenon, most scholars such as Stennes (1967), Arnott (1970) and McIntosh (1984) have given little or no attention to the nouns of the language. Several scholars' defined suffixes variously. The researcher attempts to look at suffixes based on structure, forms and grammatical functions, since suffixes are a morpheme or group of morphemes attached to an existing root or base to derive a word. Looking at suffixes and considering them as just ordinary attachment to the base forms is wrong and unrealistic. This is because the combination of suffixes attached to verbs form a single marker which distinguishes several types of verbs.

\footnotetext{
*Corresponding Author: bashtop75@gmail.com
} 


\subsection{Verbal System of Fulfulde}

The verbal system of Fulfulde shares common features with that of the nominal system (Arnott, 1970). The verb is divided into a base and a suffix. The base is the verb root; while the suffix marks tense or most appropriately it marks voice, aspect, and polarity. The base of the verb can take as many suffixes as possible and be meaningful. Some examples of suffixes go as follows:

Example 1

War-ay 'will come'

$\mathrm{rt} \quad \mathrm{cs}$

Dill-ay 'will go'

rt $\quad \mathrm{cs}$

Dogg-ay 'will run'

$\mathrm{rt} \quad \mathrm{cs}$

The suffixes in the example (a-c) above mark positive future tense of the active voice. The same verb roots, when attached to other suffixes, will derive several words with different meanings. The verbs can take as many different other suffixes as possible in order to give other several meanings.

Example 2

$$
\begin{aligned}
& \text { war-ataa 'will not come' } \\
& \text { dill-ataa 'will not go' } \\
& \text { dogg-ataa 'didn't ran' } \\
& \text { war-aay 'didn't come' } \\
& \text { dill-aay 'didn't go' } \\
& \text { dogg-aay 'didn't run' }
\end{aligned}
$$

The suffixes in the example (2a-c) above mark negative future tense of the active voice, while the suffixes in the example (2d-f) mark negative past tense of the active voice. However, some of the initial consonants of the verb roots alternate in context due to the sensitivity of the verbs to the number of the subject, but it does not affect the suffixes. Detailed explanation about Initial Consonant Alternation (ICA) are tackled by Abba (Abba,1991). Some other examples include:

Example 3

O war-ay 'he will come' > be ngar-ay 'they will come'

O dill-aay 'he didn't go' > be ndill-aay 'they didn't go'

O dogg-ataa 'he won't run' > be ndogg-ataa 'they won't run'

The examples show that the initial consonant of the verb root undergoes some phonological changes such as $w \sim n g, d \sim n d$ and $d \sim n d$, respectively. Subjects have been made plural in this case. However, like nouns, there are some verbs whose initial consonant does not alternate at all in both singular and plural subjects. Arnott (1970) describes them as 'uniform'. 


\section{Theoretical framework}

The theoretical framework adopted for the analysis of data is generative phonology (GP). Generative phonology can describe any aspect of human language, particularly the aspect of morphophonological issue. In view of this, the analysis of generativist starts by establishing the underlying systemic phoneme or phonemic representation. The concentration is on the identification of the underlying phonemes to cater for their alternation. When segments change, there are three things to consider: the affected segment, the process of its change and the condition that triggers the change (Schane, 1973 and Baba, 1998). Therefore, what changes, and how it changes are connected by an arrow pointing the direction of the change from UR to SR. As in the schema below:

$$
\mathrm{A} \longrightarrow \mathrm{B} /{ }_{-} \mathrm{C}
$$

In the rules of deletion, the (SC) is indicated by a null symbol [ø]. The segment that undergoes deletion (SD) appears to the left of the arrow and the [ø] which is the (SC) appears to the right of the arrow, represented in the rule below:

$\mathrm{A} \longrightarrow \varnothing /{ }_{-} \mathrm{B}$

\section{Verb Suffixes}

The basic form of the verb suffix is determined by the presence of an initial vowel. In some cases, the entire suffix is just a short or long vowel. Mukoshy (1991:29) considers suffixes as suffix morphemes that co-occur in most cases with the verbs and nominals. For verbs, suffixes take the tense/voice or mood morphemes as their suffix. Breedveld (1995) calls the suffixes as conjugational suffixes (cs). These suffixes are attached forms of different tense markers expressing tense, mood, aspect, discourse-related meanings (focused, focalizing) and polarity (negative and affirmative). For McIntosh (1984), these suffixes comprise of voice, aspect, and polarity (VAP). McIntosh agrees with Arnott (1970) who identifies 42 different tenses in Fula.

There are three different series of tense commonly referred to as voices, which are equally sub-divided into three: Active, Middle and Passive (AMP). Arnott (1970) argues that there are altogether 15 Active tenses, 14 Middle tenses and 13 Passive tenses. Each Active tense has middle and passive counterparts. The exception is that there is no desiderative passive, and no negative of quality for middle or passive. The conjugational suffixes have different forms for different voices. Arnott's (1970) assertion that active tense has middle and passive counterparts that contradicts verb roots selection of suffixes. In other words, while there are verb roots that can combine with all 3 voices, there are also verb roots that can combine with only one of the voices (Daudu 2005).

Voice appears to be the most important feature of verb suffixes or the conjugational suffixes as referred by Breedveld (1995) which may be the reason why she reduces the number of tenses to only two (past and present). She is careful about voice. She disagrees with conjugational suffixes being monomorphemic (monosyllabic), rather than polymorphemic (polysyllabic). Here, the researcher would choose the phonological terms mono and disyllabic to consider the morphemes than the former, which has been conveniently adopted for the study.

Like other languages, suffixes in Fulfulde are bound morphemes; therefore, they must be attached to a verb form/ verb root to produce a specific meaning. There are some verb roots 
that can take active and passive voices, while others can take only active voice, and some with only passive voice. The meaning of the voices may not be strictly classified as in the following example:

Example 4

$\begin{array}{llll} & \text { active } & \text { middle } & \text { passive } \\ \text { a. } & \text {-a } & \text {-oo } & \text {-ee } \\ \text { b. } & \text {-ii } & \text {-ake } & \text {-aama } \\ \text { c. } & \text {-(a)n } & \text {-(o)to } & \text {-(e)tee } \\ \text { d. } & \text {-aayi } & \text {-aaki } & \text {-aaka }\end{array}$

In the examples above, the entire suffixes are active, middle, and passive voices. They are categorised according to their tenses: past, present, and future tense, respectively. The suffixes of example (a) mark present tense, the ones of (b) mark past tense and the ones of (c) mark future tense, while the ones of (d) mark negative past (Arnott, 1970, and Breedveld 1995).

However, it is important to differentiate the meanings of the three voices that constitute the discussion of this study. This is because they are very important in realising the different forms of verb suffixes as well as the phonological/syntactic behaviour of the verb, as presented in the following examples:

Example 5
a. Musa yiiw-ii-ngel 'Musa bathed it (the baby)'
$\mathrm{s} \quad \mathrm{cs} \quad \mathrm{O}$
b. Musa yiiw-ake 'Musa bathed (himself)'
$\mathrm{s} \quad \mathrm{cs}$
c. Ngel yiiw-aama 'it (the child) has been bathed by.....'
$\mathrm{S} \quad \mathrm{cs}$

Example (5a) includes a subject and an object (experiencer), while (5b) has only a subject (experiencer) too, and in $(5 \mathrm{c})$ it has only the object (experiencer) occupying the subject position. Breedveld (1995) explains the three voices suggesting that the middle voice usually implies the action or process expressed by the verb that affects the subject. The verb in the middle voice of Fulfulde Maasina marks the infinitive with the suffix -aade which is represented by -aago or -aaki in the Nigerian dialects. In passive verbs, the subject of the verb is the agent of the action expressed by the verb, or the subject is the patient when the verb expresses a process. The semantic interpretation of the active, middle and passive voice markers has consequences for the argument structure of the verb.

Another feature of the conjugational suffixes is the inflection for number. This is more specific for the imperative. Thus, there are imperative singular and imperative plural. The imperative singular verb is marked by the suffix $-u$, while the plural is marked by the suffix $-e e$, as in the following examples:

Example 6

War-u (you sing.) come! 
Ngar-ee (you pl.) come!

cs

In subject inversion, there is as apparent inflection of the verb suffix in both singular and plural constructions, as represented in the following examples:

Example 7

ngar-aa 'come ( $1^{\text {st }}$ Pers. Sing. $)$ '

ngar-on 'come (2 $2^{\text {nd }}$ Pers. Pl.)

ngar-en 'come ( $1^{\text {st }}$ Pers. P1 Incl.)'

In the examples (7a-c) above, suffix -aa stands for the second person singular, requesting the person to come in isolation while -on suffix in (7b) stands for second person plural, while suffix -en stands for first person plural inclusive.

Daudu (2005) considers verb suffixes as extensions attached to root or base that result in changes in the meaning of verbs by modifying them. The extensions, for instance, -irdenotes the meaning of 'with' as in: yar-i 'to drink' and yar-ir-ii 'to drink with'. Thus, it can be regarded as a preposition. The two extensions behave in the same way; as modal -ir$/-d$-, and locative -ir-/-or- suffixes, respectively. Both occur in nouns referring to instrument, locative and place associated with the meaning of verb root for Gottschligg (2006). The extensions and suffixes are referred to as base morphemes that mark tense, voice, aspect or class number. He identifies the -d-associative, -ir- instrumental and indir- as reciprocal extension suffixes in Fula Adamawa.

Taylor (1921 and 1953) analyses verbal derivations of Adamawa Fulfulde that he considers as verbs are formed by adding certain letters to the radical/root base in which the meaning of the first form is modified. According to Taylor, there are four major verbal modifications made by adding the $-n a$, $-r a$, $-t a$, and $-d a$ modifiers/suffixes (extensions). He argues that the aspect in Fulfulde can be either completive or in-completive (perfective/imperfective). The completive takes the $-i /$ - $i$ ii suffixes while the in-completive takes the $-a /$-ay suffixes. The perfective aspect usually describes the verb, and reports a completed action as opposed to an incomplete or continuing one. Taylor presented the following examples: -na -ni, -ra -ri, $-d a-d i$ and $-t a-t i$ a. The -na modifier, which is always in transitive, gives causative form of a verb.

Example 8

$\mathrm{O}$ anndi "he knows" $\mathrm{O}$ annd- in-i-mo 'he made him know/he informed him'

O hisi 'he escaped'O his-in-i-be 'he rescued them'

However, the -ra suffix is instrumental, and it also denotes the manner in which a thing is done, as in the following examples:

Example 9

$\mathrm{O}$ narti ${ }^{1}$ 'he entered' $\mathrm{O}$ nart-ir-i 'he entered by way of'

O wurti 'he went out' O wurt-ir-i 'he went out through.....'

The third modifier presented by Taylor (1921) is the -ta- suffix that has two divisions: intensive and opposite meaning, while - $d a$ - suffix has totality and intensive meaning. The

1 The word is not found in Adamawa dialect, rather it is found in Central Northern Nigerian Dialect (Kaceccereere). 
combinations of these four extensions will produce many derived forms. These combinations can be in form of reduplication $+n a$ denoting repeated/intensive action. The combination of -ta and - $n a$ which gives -tana- extension denotes dative, for -ta and - $r a$ has -tara- which is mostly used in plural because it expresses/denotes something done in common.

Summarily, Taylor (1921) opines that each derivative verb takes a tense or mood ending as required in the same way as verbs of the first form do.

Leith-Ross (1972) identifies the $-d$ as homophonous suffix for the associative and completive verbal extensions as they occur in Fulfulde grammar. The study further presents other verbal extensions as -e associative/completive, -r- as instrumental, locative and manner suffix, adding that many nouns are formed with these elements, referring these extensions as elements.

Daudu (2002/2003) discusses structure and meaning of Fulbe personal names. In addition to analysing the cultural undertone of the Fulbe personal names, the study gives particularly a linguistic explanation of the names which include how verb suffixes determine the personal names. The following examples explain this:

Example 10

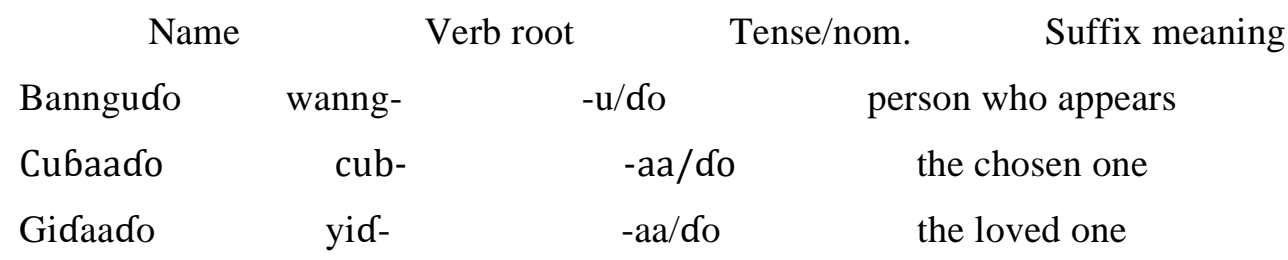

According to Daudu (2002/2003), the above examples are cases of verbo-nominals in which hybrid words consist of a verbal radical + tense suffix + a nominal suffix. The morphology of verbs in Fulfulde is that of a root + suffix which marks tense, polarity, and voice, as presented in the following examples:

Example 11

$$
\begin{aligned}
& \text { war - ii 'come' } \\
& \text { root + suffix } \\
& \text { war - aayi 'not come' } \\
& \text { root + suffix }
\end{aligned}
$$

The difference between the (11a and 11b) is simply that the former is positive, while the latter is negative. Suffixes bring about change in the initial consonant to alter the initial sound. The alternation is because of the suffixes attached onto the roots depending on the suffix following the root. The choice of either -aa- or -u- depending on the suffixes determines voice (positive or negative), aspect and polarity in the verbs.

Muhammed (2014) studies the verbal extensions of Central Northern Nigerian Dialect of Fulfulde. The study suggests two main groupings of the verbal extensions or suffixes as individual consonantal suffixes and extensions in combination. He identifies nine (9) different consonantal suffixes and some extensions in combination which include separative, reflexive, completive, compelling, intensifier and probability suffixes.

Baba (1998) discusses the morphophonological alternation in the Hausa verbal forms and identified automatic and non-automatic alternation in Hausa that are triggered by rule-based 
conditions. According to Baba (1998), phonemic variation that is conditioned by the neighbouring segment within a morpheme or across morpheme boundary is known as automatic morphophonological alternation. The non-automatic alternation changes the phonetic shape of an abstract morpheme, which are both generated by applying a morphophonological rule to the segment on UR of a word. The rule of alternation below explain this idea:

$$
\left[\begin{array}{l}
\text { +cons } \\
+ \text { back } \\
\text {-round } \\
- \text {-pal }
\end{array}\right] \rightarrow[+ \text { round }] /-\left[\begin{array}{l}
+ \text { syll } \\
+ \text { round }
\end{array}\right]
$$

\section{Example 13}

[hà $\left.{ }^{\mathrm{w}} \mathrm{urà}\right]$ be patient

[tàk ${ }^{\mathrm{w}}$ urà] sit huddled up

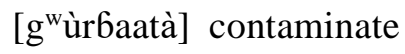

He also discussed rule of deletion as in the example below:

$$
\left[\begin{array}{l}
\text { syll } \\
\text { +low }
\end{array}\right] \rightarrow \phi /\left[\begin{array}{l}
\text {-cons } \\
\text {-syll }
\end{array}\right]_{-} \# \#
$$

Example 14

[kaw] remove

[raw] dance

[kay] take to

[kàray] loose heart

[sàray] fall over

[haw] ride

Abubakar (2000) studies the morphophonemics of the Hausa Monosyllabic Verbs. It attempts to justify critically the disyllabicity of the so-called Hausa monosyllabic verbs called 'irregular verbs.' A morphophonological study shows that some Hausa monosyllabic verbs are underlyingly disyllabic. It argues that the inflection and derivation of the verbs, $e,-r,-o$, and $-u$ suffixes attached to the verb form, undergo changes as the resultant effect derives another word. The following examples illustrate this idea:

\begin{tabular}{|c|c|c|c|c|}
\hline & Verb & -e suffix & -r suffix & -o suffix \\
\hline bi & biyee & biyar & biyoo & biyu \\
\hline $\mathrm{sa}$ & ganee & ganar & ganoo & ganu \\
\hline & fiye & - & - & fiyu \\
\hline & kiye & _ & - & kiyu \\
\hline & - & baayar & - & bayu \\
\hline
\end{tabular}

Example 15 
The examples (11a and 11b) above take the verb suffixes which show possible transformation that the verbs undergo, but (11c and 11d) take only $-e$ and $-u$ suffixes while (11e) takes only $-r$ and $-u$ suffixes, respectively that suggests the changes in the verbs.

Derivationally, the study argues that only the verbs sha, ci, ja, bi, ki, and haw becoming shayee, ciyee, janyee, biyee, kiyee, and hayee, respectively can be accounted for on the transformation it undergoes, if they are considered disyllabically at the base. However, if they fail to justify that neither anaptotic insertion of alveolar nasal /n/ nor its syncopation are convincing.

\section{Discussion}

The study dwells basically on the operations of the verb suffixes. The researcher examines verbs based on how they were derived. It seems that the VAP plays the most important role in determining the suffixes of the verbs. However, within each group of suffixes meant for a particular voice, some segments tend to exhibit a kind of morphophonological evidence in their operation.

The Fulfulde verbs are described by three parameters; viz, Voice, Aspect and Polarity (VAP). Voice of a verb can be active, middle, or passive. The aspect determines tense while polarity determines whether the verb is positive or negative.

The various suffixes for verbs depend on the aspect of verbs as well as voice. There is a set of suffixes for each voice and each suffix is a morpho semantic entity. However, the study looks at the suffixes from the phonological point view with an attempt to account for the surface form of the individual suffixes within a given set of any of the three voices. First, an attempt was made to identify the underlying suffix for each voice considering the established tense markers, as explained in the following examples:

Active voice: The underlying suffix form for the active voice is $\mathbf{u}$.

Middle voice: The underlying suffix form for the middle voice is $\mathbf{a}$.

Passive Voice: The underlying suffix form for the passive voice is ee.

From the above established voices (1,2 and 3), the individual suffixes that mark voices were identified as active voice which is -u, while middle voice has -a and passive voice has -ee. Pertaining to tenses, each voice has its own peculiar set of tense markers, as show in the following table:

Table 1.

\begin{tabular}{|l|l|l|l|l|l|}
\hline Voices/Tenses & Fut & Pres & Past & Neg. Fut. & Neg. Past \\
\hline Active-u & -(a)n & -a & -i & -(a)taa & aay \\
\hline Middle-a & -oto & -o & -ake & -(a)taako & aaki \\
\hline Passive-ee & -etee & -ee & -aama & -(a)taake & aaka \\
\hline
\end{tabular}

It can be noticed that suffixes categorically determine tense in the language. The concern therefore is tense, which has future, present and the past forms. Negativity is equally marked by the appearance of the suffixes as shown. However, what could be derived as in surface representation, which brings about the morphophonological changes of the suffixes, cannot be the same as underlying representation, as illustrated in the following examples: 


\section{Example 16}

\section{Verb roots}
a. 6orn-
b. dill-
c. dogg-
d. hokk-
e. ja6-
f. janng-
g. nyaam-
h. nyukk-
i. yar-
j. yiiw-
'to wear cloths'
'to go'
'to run'
'to give'
'to take'
'to read'
'to eat'
'to hide'
'to drink'
'to bathe'

\section{Gloss}

Considering the above verb roots, there is selectional restriction of suffixes. So, not all verb roots can take every suffix. For instance, the middle voice suffixes are restricted to specific verb roots such as 6orn-, 'to wear cloths' nyukk- 'to hide' and yiiw 'to bathe'. The verb root yiiw- 'bathe' can take all suffixes that determine some changes in the aspects of the voices discussed above. The following examples are related to active voice:

Example 17

\section{Active Voice: UR}
a. /yiiw-u/ 'bath (someone)'
b. /yiiw-u-n/ 'will bath (someone)'
c. /yiiw-u-a/ 'to bath'
d. /yiiw-u-i/ 'has bathed (someone)'
e. /yiiw-u-aay/ 'did not bath (someone)'
f. /yiiw-u-taa/ 'will not take bath'

\section{Active Voice: SR}

[yiiw-u] 'bathe(someone)'

[yiiw-an] 'will bath (someone)'

[yiiw-a]'to take bath'

[yiiw-i] 'bathed (someone)'

[yiiw-aay] 'hasn't bathed...'

[yiiw-ataa] 'will not bath (someone)'

Looking at example (17) above, the proposed underlying verb suffix for active voice in the language is $[u]$, but it either alternates to a different segment or it is deleted in the surface form. The verb is inflected according to the tenses mentioned earlier as triggers of the alternation with restriction to a particular voice. The $[\mathrm{u}]$ sound in the underlying form is considered to be alternating or deleted according to the features of the adjacent segment of the suffix in the surface form.

Looking at the table above, one can notice the tense markers for the active voice as: $-\boldsymbol{n}$ for future, $-\boldsymbol{a}$ for present tense, $\boldsymbol{- i}$ for past tense, a ay for negative past tense, and $\boldsymbol{t a \boldsymbol { a }}$, for negative future tense. Example (17) demonstrates both the underlying and surface representations of the $[\mathrm{u}]$ sound that is considered as basic. Therefore, the concern is how to justify the rule-based alternation of the $/ \mathrm{u} /$ sound where $/ \mathrm{u} />[-\mathrm{a}]$ as in $/ \mathrm{jr}: \mathrm{w}-\mathrm{an} /$, and $/ \mathrm{jr}$ :wata:/, while $/ \mathrm{u} />[\varnothing]$ as in /ji:w-a/, /ji:w-I/, and /ji:w-aaj/. A look at the following example explains the alteration rule. 
$\left|\begin{array}{c}+ \text { back } \\ + \text { high } \\ + \text { round }\end{array}\right| \rightarrow\left[\begin{array}{c}\text {-back } \\ + \text { low }\end{array}\right] /[$ +alveolar $]$

Thus, the rule of alternation asserts that $/ \mathrm{u} /$ becomes [a] before an alveolar sound, as presented in the following example.

Example 18

a. /ji:w-u-n/ > [ji:w-a-n] 'yiiwan 'will bathe'.

b. /yiiw-u-taa/ > [yiiw-ataa] 'will not bath (someone)'

However, in some cases such as active voice tense marker $/ \mathrm{n} /$, the negative future tense markers active /taa/, middle /taako/ and passive /taake/, etc. may also be preceded by a vowel, e.g. (active /(a)taa/, middle /(a)taako/ and passive /(a)taake/, in which the rule of deletion should be resorted instead of alternation.

Example of rule of deletion.

$$
\left|\begin{array}{l}
+ \text { back } \\
+ \text { high } \\
\text { +round }
\end{array}\right| \longrightarrow[\varnothing] /-[+ \text { syll }]
$$

Here, the rule asserts that $/ \mathrm{u} /$ becomes $[\varnothing]$ before syllabic sounds, as presented in the following example.

Example 19
a. /jr:w-u-a/ > [ji:w-a] yiiwa 'bath'.
b. /yiiw-u-i/ > [yiiw-i] 'bathed (someone)'
c. /yiiw-u-aay/ > [yiiw-aay] 'hasn't bathed...'

From the examples provided above, the roots of the words remain unchanged with the various suffixes attached to roots in deriving many different words of different meanings. However, the morphophonological conditions of suffixes (the changes, the suffixes undergo) are responsible for the derived words.

However, as discussed earlier under active voice, the verb root yiiw- 'bathe' can take many suffixes that determine some changes in the aspects of the voices. Examples of the middle voice include:

Example 20

\section{Middle Voice: UR}
a. /yiiw-a/ 'take bath'
b. /yiiw-a-oto/ 'will take bath'
c. /yiiw-a-o/ 'is taking bath'
d. /yiiw-a-ake/ 'has taken bath'
e. /yiiw-a-aaki/ 'has not taken bath'
f. /yiiw-a-ataako/ 'will not bath'

\section{Middle Voice: SR}

$$
\begin{aligned}
& \text { [yiiw-a] 'take bath' } \\
& \text { [yiiw-oto] 'will take bath' } \\
& \text { [yiiw-o] 'is taking bath' } \\
& \text { [yiiw-ake] 'has taken bath' } \\
& \text { [yiiw-aaki] 'has not taken bath' } \\
& \text { [yiiw-ataako] 'will not bath' }
\end{aligned}
$$

Considering the table above, tense markers for the middle voice are -oto for future, -o for present tense, -ake for past tense, -aaki for negative past tense, and ataako, for negative 
future tense. Example (20) demonstrates both the underlying and surface representations of the /a/ sound that is considered as the basic. Therefore, the concern is how to justify the rule-based deletion where /a/ > [ø] as in /ji:w-oto/, / ji:w-o/, /ji:w-ake/, /ji:w-a:ki/, and /jI:w-ata:ko/. Consider the rule of deletion below.

$$
\left|\begin{array}{c}
+ \text { cent } \\
+ \text { low } \\
\text {-round }
\end{array}\right| \longrightarrow[\varnothing] /[+ \text { syll }]
$$

Thus, the rule asserts that $/ \mathrm{a} /$ becomes $[\varnothing]$ before a syllabic sound. Consider the following example:

Example 21
a. /yiiw-a/ > [yiiw-a] 'take bath'
b. /yiiw-a-oto/ > [yiiw-oto] 'will take bath'
c. /yiiw-a-o/ > [yiiw-o] 'is taking bath'
d. /yiiw-a-ake/ > [yiiw-ake] 'has taken bath'
e. /yiiw-a-aaki/ > [yiiw-aaki] 'has not taken bath'
f. /yiiw-a-ataako/ > [yiiw-ataako] 'will not bath'

From the examples provided above, the roots of the words remain unchanged with the various suffixes attached to the roots in deriving many different words of different meanings. But the morphophonological conditions of the suffixes (the changes, the suffixes undergo) are responsible for the derived words.

In the case of passive voice, the verb root yiiw- 'bathe' can take many suffixes that determine some changes in the aspects of voices. Therefore, below are some examples of the passive voice.

\section{Example 22}

\section{Passive Voice: UR}
a. /yiiw-ee/ 'to be bathed by'
b. /yiiw-ee-etee/ 'will be bathed'
c. /yiiw-ee-ee/ 'is being bathed'
d. /yiiw-ee-aama/ 'has been bathed'
e. /yiiw-ee-aaka/ 'has not been bathed'
f. /yiiw-ee-ataake/ 'will not be bathed'

\section{Passive Voice: SR}

$$
\begin{aligned}
& \text { [yiiw-ee] 'to be bathed by' } \\
& \text { [yiiw-etee] 'will be bathed' } \\
& \text { [yiiw-ee] 'is being bathed' } \\
& \text { [yiiw-aama] 'has been bathed' } \\
& \text { [yiiw-aaka] 'has not been bath' } \\
& \text { [yiiw-ataake] 'will not be bathed' }
\end{aligned}
$$

Tense markers for the passive voice are -etee for future, -ee for present tense, -aama for past tense, -aaka for negative past tense, and atake, for negative future tense. So, example (22) demonstrates both the underlying and surface representations of the /ee/ sound that is considered as the basic. The rule-based deletion is built as follows /ee/ > [ø] as in /j1: w-

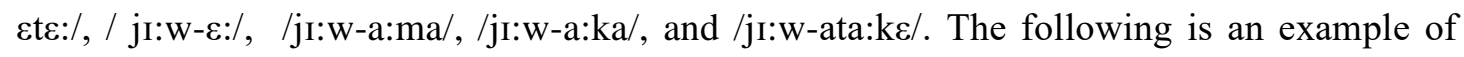
deletion

$$
\left|\begin{array}{l}
+ \text { front } \\
+ \text { mid } \\
\text {-round }
\end{array}\right| \longrightarrow[\varnothing] /-[+ \text { syll }]
$$


+temse

Thus, the rule asserts that /ee/ becomes [ $\varnothing]$ before a syllabic sound, as in the following example.

Example 23
a. /yiiw-ee/ > [yiiw-ee] 'to be bathed by'
b. /yiiw-ee-etee/ > [yiiw-etee] 'will be bathed'
c. /yiiw-ee-ee/ > [yiiw-ee] 'is being bathed'
d. /yiiw-ee-aama/ > [yiiw-aama] 'has been bathed'
e. /yiiw-ee-aaka/ > [yiiw-aaka] 'has not been bath'
f. /yiiw-ee-ataake/ > [yiiw-ataake] 'will not be bathed'

From the examples of the passive voice provided above, the roots of the words remain the same with the various suffixes attached to them to derive different words of different meanings. But the morphophonological conditions of the suffixes (the changes, the suffixes undergo) are responsible for the derived words.

\section{Conclusion}

This paper attempts to explain the changes of suffixes within a verb and considers its form and behaviour. The paper discovers that both morphology and phonology interact in the process of changes that occur within the verbs in Fulfulde. It argues that each voice has a single underlying suffix, which interacts with various tense suffixes to derive a complete verbal complex. However, the study proves that although the surface representations of the various suffixes are many, there are only two conditions that trigger their changes (i.e. alternation and deletion rules).

Considering the three voices, i.e. the active, middle and the passive voices, as demonstrated in the analysis above, two different rules are involved in all. In the case of active voice, alternation and deletion rules are identified, while in the case of middle and passive voices only deletion rule is applicable. In the active voice, where $/ \mathrm{u} /$ alternates to [a], adjacency to an alveolar sound was identified as the common trigger, and where /u/ becomes null $[\varnothing]$, adjacency to a syllabic sound was identified as the common trigger. Moreover, in the case of middle and passive voices, where the underlying /a/ becomes null $[\varnothing]$ in the middle voice and /ee/ becomes $[\varnothing]$ in the passive voice, adjacency to a syllabic sound was also identified as the common trigger in both. Considering the various environments that have different suffixes with common characteristics across the three voices, only two rules are postulated to capture them. 


\section{Acknowledgment}

Acknowledgment and/or disclaimers should appear before References. Names of funding organizations should be written in full. The authors are advised to describe author contributions, e.g., as follows: S.A. gave the idea, F.A. did the experiments, F.A. and S.A. interpreted the results, S.A. wrote the paper.

\section{References}

[1] Abba, M. (1991). "Initial Consonant Permutation in Fulfulde Verbs and Nouns". In Abba, I. A., Mukoshy, I. \& Tahir, G. (Eds.) (1991). Studies in Fulfuld Language, Literature and Culture. Kano: The Centre for Study of Nigerian Languages.

[2] Abubakar, A. (2000). An Introductory Hausa Morphology. Department of Languages and Linguistics, University of Maiduguri, Nigeria.

[3] Abu-Manga, A. (1991). Length and Focus in Fulfulde Verbal System. In Abba, I. A., Mukoshy, I. and Tahir, G. (Eds.) (1991). Studies in Fulfulde Language, Literature and Culture. Kano: The Centre for Study of Nigerian Languages.

[4] Arnott, D. W. (1970). The Nominal and Verbal Systems of Fula. London: Oxford University Press.

[5] Baba, A. T. (1998). The Morphophonological Alternations in the Hausa Verbal

[6] Forms. (Berichte des Sonderforschungsbereichs 268.Vol. 12). Frankfurt.

[7] Breedveld, J. O. (1995). Form and Meaning in Fulfulde: A Morphophonological Study of the Maasinankoore, Leiden Research School CNWS.

[8] Daudu, G. K. (2002/2003). "The Structure and Meaning of Fulve Personal Names". In: Harsunan Nijeriya. Vol. XX, pp. 75 - 90. Centre for the Study of Nigerian Languages, Bayero University, Kano. Kano - Nigeria. (2005). A Study of Movement Operations in Fulfulde. Ph D. Thesis, University of Maiduguri.

[9] Gottschligg, P. (2006). "Elaboration and Simplification in Fula Verbal Morphology". In: Bernard Carom and Petr Zimz (eds.) Sprachbund in thw West African Sahel. LLACAN-CNRS and Faculty of Humanities, Charles University Prague.

[10] Hyman, L. M. (2007). Phonology: Theory and Analysis, Holt, Rinehart and Winston. Leith-Ross, (1921). Fulani Grammar. Lagos and London.

[11] Matthews, P. H. (2007). Oxford Concise Dictionary of Linguistics. Oxford: Oxford University Press.

[12] McIntosh, M. (1984) Fulfulde Syntax and Verbal Morphology, Great Britain: St Edmuds Bury Press Ltd.

[13] Mohanan, K. P. (1982). 'Lexical Phonology'. Ph.D. Thesis Submitted to the Department of Linguistics and Philosophy, Massachusetts Institute of Technology.

[14] Mukoshy, I. (1991). A Review of the Fulfulde Classes. In: Abba, I. A., Mukoshy, I. and Tahir, G. (Eds.) (1991). Studies in Fulfulde Language, Literature and Culture. Kano: The Centre for Study of Nigerian Languages. (1977). "Fulfulde Suffix Morphophonemic Changes". Harsunan Nijeriya. Vol. VII, pp. 1-22, Centre for the Study of Nigerian Languages. Bayero University Kano. Kano, Nigeria.

[15] Schane, S. A. (1973) Generative Phonology, New Jersy: Eagle wood cliffs.

[16] Taylor, (1921). A Grammar of Adamawa Dialect of the Fulani Language. OUP.

[17] Taylor, (1953). A Grammar of Adamawa Dialect of the Fulani Language. (2nd edition) OUP. 